\title{
How Much EDS Resolution do I Need?
}

\author{
N. A. Bulloss
}

Thermo Fisher Scientific, 5225 Verona Rd, Madison, WI 53711, USA

Energy-dispersive spectrometry (EDS) is a staple analytical technique in most electron microscopy laboratories. EDS detectors collect and display a spectrum of all x-ray energies simultaneously. It is a powerful technique enabling the operator to rapidly and accurately determine the chemical composition or the distribution of elements and phases within a sample. Common EDS data collection techniques are; quantitative and qualitative point analyses, qualitative and quantitative $\mathrm{x}$ ray maps and linescans. Another increasingly important use of EDS data is the automatic determination of chemical phase distribution in samples using multivariate statistical analysis applied to the EDS data from all of the map pixels.

Typical EDS detector spectral resolutions (usually defined by the Mn Ka FWHM) range from approximately $120 \mathrm{eV}$ to $150 \mathrm{eV}$. This is in contrast to the complementary technique of wavelengthdispersive spectrometry (WDS) whose spectral resolutions are approximately an order of magnitude better than EDS depending upon the chosen crystal/energy range.

Modern silicon drift detector (SDD) energy-dispersive spectrometers have significantly improved upon the older lithium-drifted silicon (SiLi) crystal designs, enabling analysts to obtain significantly higher stored x-ray count rates with little compromise in resolution (FIG. 1).

To take advantage of the power of the SDD detectors these EDS systems can be run at very high $\mathrm{x}$ ray count rates, thus reducing analysis times and maximizing sample throughput. This is particularly important in the typical busy laboratory environment where SEM time is valuable. Under these typical high count rate conditions the system is not operating at the maximum (specification) resolution. As continuous improvements are being made to SDD EDS systems the spectral resolution is being improved in small increments. However, the highest resolution detectors often come at a higher price relative to lower resolution detectors prompting the question from EDS customers; "How much EDS resolution do I need".

When operating EDS systems analysts need to know, with confidence, that the peak identifications are accurate, particularly where peak overlaps occur. Table 1 shows a few of the typical peak overlaps encountered in the materials science and biological sciences fields. The first column lists the overlapping elements and peaks. The second column shows the difference (in eV) between the overlapping peaks. The third and fourth columns show the resolution of the detector at the energy of the peak overlap for detectors with a $\mathrm{Mn} \mathrm{K \alpha}$ resolution of 130 and $120 \mathrm{eV}$. It is clear from the table that in none of these examples does an improvement in detector resolution from $130 \mathrm{eV}$ to $120 \mathrm{eV}$ completely resolve any of the peak overlaps. The EDS resolution is still well below the $\Delta \mathrm{eV}$ of the overlapping peaks. The peaks might look a little more resolved on the screen but what is seen on the screen is a visual representation of the data and does not affect the quality of the peak deconvolution. At the end of the day a robust peak deconvolution algorithm is the only way to accurately resolve the overlapped peaks. The quality of the deconvolution routine becomes increasingly important if the data is being used in quantitative analysis. The original peak deconvolution algorithms were written 
when typical detector resolutions were on the order of $160 \mathrm{eV}$ and worked well at those resolutions. The algorithms therefore work extremely well at detector resolutions of $150-120 \mathrm{eV}$. Therefore, in real world applications, there is no practical improvement in analytical results with a $120 \mathrm{eV}$ detector over a $130 \mathrm{eV}$ detector.

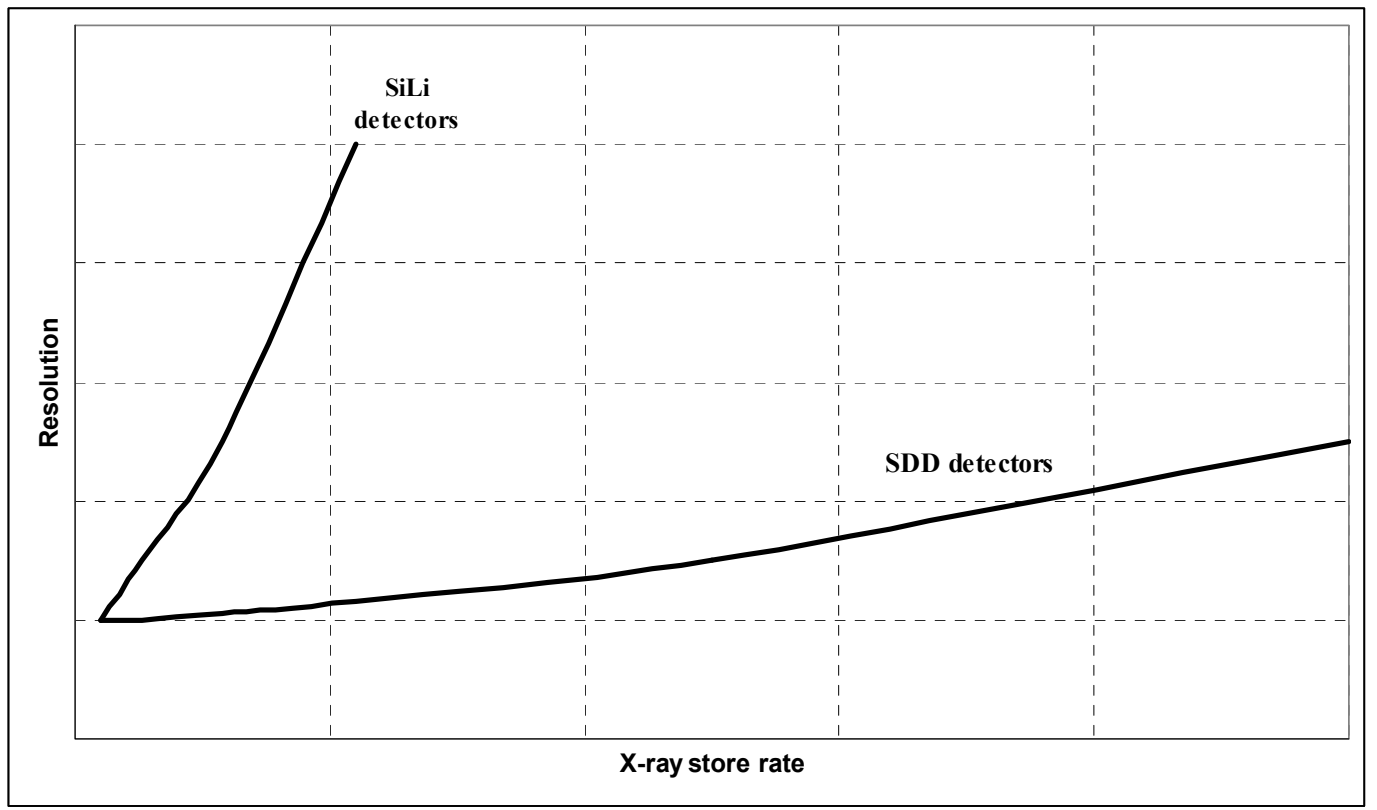

FIG. 1. A comparison of SiLi and SDD detector resolution performance vs. the output x-ray count rates. SDD detectors can be operated at much higher x-ray count rates without significantly compromising resolution.

TABLE 1. Examples of peak overlaps typically encountered in material science and biological samples.

\begin{tabular}{|c|c|c|c|}
\hline Peak overlaps & Peak overlap $\Delta \mathbf{e V}$ & $\begin{array}{c}\text { Peak resolution (at } \\
\text { Mn Ko } \boldsymbol{\alpha} \mathbf{1 3 0 e V})\end{array}$ & $\begin{array}{c}\text { Peak resolution (at } \\
\text { Mn Ko } \boldsymbol{\alpha} \mathbf{1 2 0 e V})\end{array}$ \\
\hline $\mathrm{Na}(\mathrm{K} \alpha), \mathrm{Zn}(\mathrm{L} \alpha)$ & 29 & 173 & 154 \\
\hline $\mathrm{Mo}(\mathrm{L} \alpha), \mathrm{S}(\mathrm{K} \alpha)$ & 15 & 182 & 164 \\
\hline $\mathrm{P}(\mathrm{K} \alpha), \mathrm{Zr}(\mathrm{L} \alpha)$ & 28 & 180 & 162 \\
\hline $\mathrm{Os}(\mathrm{M} \alpha), \mathrm{P}(\mathrm{K} \alpha)$ & 104 & 180 & 162 \\
\hline $\mathrm{Ti}(\mathrm{L} \alpha), \mathrm{O}(\mathrm{K} \alpha)$ & 73 & 169 & 150 \\
\hline $\mathrm{Al}(\mathrm{K} \alpha), \mathrm{Br}(\mathrm{L} \alpha)$ & 7 & 177 & 158 \\
\hline
\end{tabular}

